\title{
Use of a Blockchain-Based Marketplace to Incentivize Participation in Virtual Clinical Trials
}

\author{
Phillip Olla, Audacia Bioscience, Windsor, Canada \\ Mustafa Taher Abumeeiz, Audacia Bioscience, Windsor, Canada \\ Lauren Kay Elliott, Audacia Bioscience, Windsor, Canada \\ Vijay Rajasekar, Audacia Bioscience, Windsor, Canada \\ Stephen Bartol, Audacia Bioscience, Windsor, Canada
}

\begin{abstract}
There is an emerging need for advancements in how clinical trials are conducted given the global pandemic the world is facing. Healthcare institutions are moving towards using digital technologies to avoid physical interactions between doctors and clinical trial participants. However, difficulties in recruiting and retaining participants are still prevalent. To overcome this issue, an incentive system that can be trusted by doctors as well as trial participants is required. The authors present a detailed report of Cashish, a blockchain-based incentivization system that rewards trial participants in the form of cryptocurrency tokens that they can utilize in an online marketplace that is also backed by the same blockchain. Usage of blockchain technology to provide research participation incentives eliminates the need for trust systems and ensures transparency between doctors and clinical trial participants, while ensuring participant anonymity.
\end{abstract}

\section{KEYWORDS}

Cannabis Research, Cryptocurrency, Ethereum, Participant Recruitment, Smart Contracts

\section{INTRODUCTION}

Blockchain systems were first introduced to manage cryptocurrency transactions such as Bitcoin (Maslove et al., 2018). As such, they provide a secure basis for online contracts, wherein participants can sign off on certain actions with another source (Nugent et al., 2016). These systems lend themselves to being applied beyond direct financial exchanges and can serve a role in incentivization programs (Maslove et al., 2018; Shrestha and Vassileva, 2018). When applied to clinical trials, these systems can utilize Blockchain to secure data and increase participant recruitment and/or retention (Benchoufi and Ravaud, 2017). The purpose of this paper is to establish the benefit of using a Blockchain-based incentivization system, in this case, Cashish virtual tokens, to increase participation, retention, and facilitate secure medical data exchange in Virtual Clinical Trials.

DOI: 10.4018/JTA.20210101.oa4

This article published as an Open Access article distributed under the terms of the Creative Commons Attribution License (http://creativecommons.org/licenses/by/4.0/) which permits unrestricted use, distribution, and production in any medium, provided the author of the original work and original publication source are properly credited. 
Virtual Clinical Trials (VCTs), also called remote or decentralized trials, are a relatively new method of conducting clinical research taking full advantage of technologies such as mobile apps, electronic monitoring devices, and online social engagement platforms (National Academies of Sciences, Engineering, and Medicine (NASEM), 2019). VCTs may overcome the challenges faced in conventional clinical trials wherein it is expensive to recruit even a limited number of suitable participants (Huynh et al., 2014). In traditional clinical trials participants are recruited through hospital visits, medical clinics, or using media such as newspaper/radio/television ads. The reach and ability to participate are often limited by participants' geography.

The COVID-19 pandemic has invigorated the growth of VCT adoption for both drug development and treatment therapies. VCT tools are creating a new era of speed, efficiency, and transparency to advance clinical studies, supporting the accelerated development of COVID-19 vaccines (van Dorn, 2020). The COVID-19 pandemic is fast-tracking the transition to more virtual approaches for gathering remote clinical data, which facilitates new advances and provide more convenient options for patients participating in studies.

In VCTs a combination of traditional participant recruitment and digital recruitment is used. The combination of recruitment strategies leads to more rapidly reaching the enrollment target resulting in faster initiation of the study (NASEM, 2019). When occuring digitally, participants are targeted directly via web-based platforms, such as search engines and social media. This approach allows for fast recruitment of participants without geographical limitations, reaching eligible trial participants worldwide.

A broader digital recruitment strategy targeting participants across geographical locations results in a more diverse group of study participants. This increasingly diverse pool of participants would mean a higher degree of representation of the real-world than what can be obtained in traditional clinical trials.

Additionally, VCTs have the potential to address issues regarding participant recruitment for research into sensitive topics. Topics such as alcohol, cannabis, or sex and sexuality can become difficult to investigate due to participant concerns around confidentiality and anonymity (Heath et al., 2018; McCormack, 2014). Perceived social taboos may also lead participants to be less willing to participate in research on said sensitive topics for fear of persecution. Through a clinical trial incentive system using blockchain technology, participants have the opportunity to participate in studies or surveys that they qualify for virtually and anonymously.

The VCT platform and it's use of a concept called dynamic consent lends researchers control over areas of research that are otherwise difficult to navigate. For example, the VCT platform allows researchers to maximize participants recruited to studies, to control how data can be used in ongoing research, and to give participants the opportunity to continue earning rewards for use in an online marketplace. Dynamic consent ensures that research participants can maintain full control of the data that they send to research companies while at the same time giving companies the opportunity to increase their use of collected data. By maintaining consistent email communication with participants, dynamic consent allows research companies to send requests for data to participants while giving participants their control of consent via email. These requests do not have to be for new data but can seek to apply existing data the participant has already given to new studies. The potential for this to expand the reach of meta-analyses and other research endeavors with complex data requirements is great. Dynamic consent enables researchers to obtain consent to use data collected from participants in multiple different contexts while still ensuring that data is protected, patients are informed of this usage, and can decline if they so wish. In the context of this research platform, dynamic consent will be used to maximize the participants recruited to studies and to continually use their data in future studies, adding any missing information or new variables for analysis if required.

However, it is also equally important to retain recruited participants to follow-up and complete subsequent research requirements. Kadam et al. (2016) identified various challenges in patient recruitment and retention. Their research showed that changes in participants' physical residence 
and poor compliance with study protocol had a moderate effect on subject retention. They also recommended retention strategies that involve flexible appointment times, better education about clinical trials for participants, and financial incentivization.

Virtual Clinical Trials solve retention challenges related to participants' convenience. Since the primary method of delivery of research questionnaires is through electronic media, participants have a higher degree of flexibility in their participation in the trials. Ali et al. (2020) conducted a virtual clinical trial and detailed how they achieved a $96 \%$ retention rate through the use of digital questionnaire distribution methods and non-financial incentives. However, financial incentivization of participation still remains a key challenge. Key factors include ethical considerations, methods of incentivization, and trustworthy incentive distribution methods.

To address some of the concerns with rewarding clinical trial participants, specifically to eliminate the need for a trust system between doctors and participants, blockchain technology can be used. This paper is structured as follows: it first introduces concepts and platforms that are discussed throughout such as virtual marketplaces and cryptocurrencies. It then provides a detailed discussion about the technology systems used in the Blockchain-based incentivization for participation in VCTs. Topics such as smart contracts, Ethereum, and privacy and security of participant data are also discussed. Prior to the conclusion the paper provides a discussion about the findings and future research.

\section{BACKGROUND}

\section{Electronic Marketplaces}

An electronic marketplace can be defined as a platform for regulated transactions and exchanges (Wang and Archer, 2007). In more commerce-oriented terms, a marketplace can also be defined as a position in the supply chain which acts as an intermediary between buyer and seller. While there is no one unified definition of a cryptocurrency, most cryptocurrencies can be described as a unit of exchange like any other currency, but with more digital options for transactions (Maese et al., 2016). Cryptocurrencies are typically untied to banks or governments, providing value outside of any one nation's sovereignty. Marketplaces can utilise cryptocurrency to exchange funds between persons or institutions. If these marketplaces are then linked to other activities, in the case of this paper, VCTs, the activities can be incentivized through exchanges on the platform, such as with cryptocurrency (Maslove et al., 2018). For the purposes of this paper, the Cashish platform will be examined in terms of its potential functionality for incentivization for participation in VCTs. Described in more detail later, the Cashish marketplace hopes to incentivize participants with opportunities to earn cryptocurrency by registering for specified studies.

\section{Challenges of Cannabis Research}

In recent years, innovative studies have been published globally showing a high potential for the efficacy and safety of cannabis products as a treatment for new indications (Nutt et al., 2021). With the legalization of cannabis, more medical studies and clinical trials are being mandated to explore the physiological, neurological, and psychological effects of its active substances. Cannabis research needs to be conducted using a preclinical and clinical trial framework, yet this approach is very expensive (U.S. Department of Health and Human Services (USDHHS) et al., 2014). The average drug clinical trial cost for an individual pharmaceutical company or sponsor is approximately $\$ 1-7$ million dollars for Phase I, \$7-20 million dollars for Phase 2, and \$10-50 million dollars for Phase 3 . The cannabis industry does not have the financial influence of the pharmaceutical sector, and although cannabis is being used for medical therapeutic purposes, the rigor typically applied to a clinical drug trial is not being applied.

All key cannabis stakeholders including medical, legislators, cannabis-licensed producers, pharmaceutical companies, and consumer groups agree that more knowledge is needed regarding 
the therapeutic use of cannabis. To help fill this gap, a significant increase in scientific research is required (Schlag et al., 2021). The current gold standard for therapeutic knowledge generation is via clinical trials within the clinical setting. A new approach that is being proposed is pragmatic clinical trials that take the trials into the community using a crowdsourcing model. Crowdsourcing cannabis data could provide a route to a better understanding of cannabis. Some of the advantages of crowdsourcing include gathering data rapidly at a very low cost and using research subjects that are willing to participate for incentives that are relatively inexpensive compared to the traditional clinical trial costs of $\$ 200$ - $\$ 500$. To date, there is no scientific crowdsourcing cannabis research data via clinical trials. The approach presented here ensures data is secure, anonymized, and research participants can be compensated with a currency that is anonymous, valuable, and usable.

\section{Blockchain in Healthcare}

A blockchain is a decentralized network where each node contains a synchronized copy of a ledger that records transactions between members of the network (Zheng et al., 2017). It was first introduced in 2008 (Nakamoto, 2008) as a means to implement a decentralized digital currency called Bitcoin. Blockchains can be used to handle transactions between parties that do not trust each other. Therefore, they are also referred to as trustless networks.

Blockchains have become extremely popular in recent years mostly due to the hype behind Bitcoin. However, industry leaders across various domains such as healthcare, finance, supply chain management, etc., have used blockchains to replace existing systems that required central authorities and middlemen, thereby decentralizing and accelerating time-critical processes with great success (Monrat et al., 2019).

\section{Cryptocurrencies as Research Incentives}

Before discussing blockchain technology in-depth, it is important to address the concept of cryptocurrencies, which form the core reason behind a blockchain's functionality as well as its popularity. A cryptocurrency is a decentralized digital currency that can be sent to or received from a member on the blockchain network (Dinh et al., 2018). Here, it is important to note that there is no direct transfer of assets or data that takes place between the members. Rather, the blockchain software keeps track of the amount of cryptocurrency held by the members of the network and increases/ decreases the amount when it recognizes and acknowledges that a transaction has occurred. This is why blockchains are referred to as distributed ledgers.

Cryptocurrencies have a monetary value based on real life assets associated with them, such as the US Dollar. Cryptocurrencies quickly became popular among tech enthusiasts due to their promise of zero governance, complete decentralization of information, and transparency while maintaining anonymity (Monrat et al., 2019). Cryptocurrencies are a finite resource and the extraction of this resource depends on being a participating member of the network. Since the supply is depleted as more people join the network, the value of the smallest unit of a cryptocurrency will keep increasing. This leads to more people joining the network and the value of the cryptocurrency will compound exponentially.

The popularity of the first cryptocurrency, Bitcoin, has led to several other implementations of cryptocurrencies. There are currently over 5000 other cryptocurrencies, or AltCoins, available worldwide (Redman, 2020).

The platform described in this paper utilized these features of blockchains to incentivize distribution of clinical trials. Trial participants are rewarded using custom cryptocurrency that can be kept track of using a distributed online ledger so that participant actions and rewards can be kept track of and records not tampered with. This ensures trust among all users of the system. A clinical trial reward system/marketplace that utilizes blockchains as a platform for reward distribution is built using smart contracts and is therefore referred to as a blockchain-based application or a Decentralized Application (DApp). 
One of the most important aspects of clinical trials is the issue of always ensuring privacy and security. Blockchain systems use asymmetric cryptography to secure transactions between the clinical trial platform and research participants. This provides an increase in security benefits and protects from potential hackers or malicious users. Public keys can be shared with other users' in marketplaces, as they do not give any information regarding personal data, clinical samples, or biometric data collected.

\section{Blockchain-Based Applications and Smart Contracts}

Applications developed using blockchain frameworks to satisfy a business case are referred to as blockchain-based applications (Casino et al., 2019). Blockchain-based applications are built using a collection of smart contracts. A smart contract is a code representation of a real-life contract formed between parties that want to engage in a business negotiation (Mohanta et al., 2018). The results of this negotiation can be the transfer of cryptocurrency or other digital assets.

One of the major milestones in the development of blockchain-based applications was the introduction of Ethereum (Buterin, 2013). Ethereum provides a Turing-complete programming language named Solidity that allows developers to write smart contracts, deploy them on the blockchain, and use functionality from these deployed smart contracts to satisfy real life use cases (Almeida et al., 2018; Casino et al., 2019; Macdonald et al., 2017). It also provides the Ethereum Virtual Machine (EVM), an isolated sandbox for the execution of compiled smart contracts. Ethereum software is available in many popular languages such as Go, Java, Python etc. The go-ethereum client, otherwise referred to as Geth, is the most used and well-maintained implementation of the Ethereum protocol.

The Ethereum protocol, otherwise known as ERC-20, can be applied to custom cryptocurrencies. ERC-20 tokens function as blockchain-based assets. They are generated through the Ethereum network, carry value, and can be transferred between holders (Somin et al., 2018). One major difference between ERC-20 tokens and Bitcoin/other prominent cryptocurrencies is that they do not run on their own blockchains (Somin et al., 2018). Over 200,000 ERC-20-compatible tokens are available on Ethereum's main network, with over 160,000 of these being completely compliant with ERC-20 standards (Chen et al., 2020; Di Angelo and Salzer, 2020). A list of rules which all fungible Ethereum tokens need to comply with has been defined by ERC-20 (Ethereum, 2021).

The ERC-20 token enables developers to predict how newly-created tokens will function as a part of the Ethereum system (Somin et al., 2018). This allows developers to work with Ethereum knowing that as long as the token they create follows the standards set out by ERC-20, new projects can proceed without the need to be reworked as new tokens are released (Fenu et al., 2018). It also ensures that new tokens will function seamlessly with other tokens available on the Ethereum platform. Altogether, the ERC-20 standard facilitates the cohesive performance of different types of Ethereum tokens within the system (Chen et al., 2020). Most of the digital wallets which support Ether, the currency native to Ethereum, support tokens compliant with ERC-20 standards (Fenu et al., 2018).

While Bitcoin and Ethereum are blockchains, Hyperledger presents itself as a common hub for various blockchain platform development-related projects (Dinh et al., 2018). It was founded in 2015 by The Linux Foundation, with a primary focus on approaching the development of blockchain platforms from an enterprise perspective. It is a collection of standards, tools, and projects that enable the development of private blockchains for businesses (Casino et al., 2019). Hyperledger provides Fabric, a modular blockchain framework that an organization looking to develop a blockchain-based business platform can use. It enables Smart Contract development in well known programming languages such as Javascript and Python, which eliminates the need for developers to learn a new language like Solidity. It supports only permissioned blockchain networks.

Ethereum or Hyperledger platforms can be leveraged to build the clinical trial reward system. However, both platforms have their advantages and disadvantages (Dinh et al., 2018). To provide some background for this decision, a high-level implementation of Cashish, a blockchain-based marketplace, will be described. 


\section{METHODS}

A blockchain enabled mobile wallet was created for research participants to collect virtual points for participating in research. Engaging in research activities generated smart contracts, and when the research activity was completed within a specified timeframe, rewards were available in a virtual wallet. A Metamask wallet was created to securely hold incentives research participants received in a proprietary points system called Cashish. In this study, Cashish was the digital currency, and could be classified a cryptocurrency as it was available in the Metamask platform. Cashish points were awarded based on the type of research tasks completed. Examples include surveys, clinical trials, sharing stories, or product feedback. The points awarded were deposited in the wallet and could be redeemed within the marketplace for $2 \mathrm{x}$ the value or the value could be transferred to other cryptocurrencies such as Ethereum using online exchanges.

A web application that researchers and participants could access to complete research requirements was created using open-source technology applications on the Wordpress and WooCommerce platforms. This was referred to as the Cashish marketplace portal. The Cashish platform refers to the complete architecture of the blockchain-based marketplace and the associated reward system. Cashish Points, the primary cryptocurrency of the rewards platform, were implemented using the ERC-20 protocol. This protocol is necessary for smart contracts created on the Ethereum blockchain using token implementation and outlines rules which all tokens based in the Ethereum platform must follow. The standards set by the ERC-20 outline how tokens can be transferred, the approval process for transactions, and the process for users to access token-related data.

Blockchain smart contract functionality was used within the marketplace to encourage research participants' engagement in transactions within the marketplace. Transactions were conducted between sponsoring brands, strategic partners, or with other research participants. Research participants with Cashish points could gain access to goods and services using the points they earned by participating in research through a smart contract transaction. These digitally signed agreements prevented any kind of fraud and eliminated third party payment processing fee(s). The blockchain platform of choice had to support automated transactions based on predetermined conditions or triggered events.

\section{RESULTS}

\section{Comparisons Between Blockchain Platforms}

There are numerous blockchain platforms and technologies. Selecting the platform right for the task is important in ensuring the success of a blockchain project. Ethereum and Hyperledger both provided robust smart contract development platforms that could help achieve the requirements for the marketplace prototype mentioned above. However, the following was observed when comparing the two platforms. Hyperledger Fabric is more than a blockchain platform. It aims to solve common issues that arise when developing blockchain solutions for enterprise grade systems. However, this also means that a lot of development effort is required to develop solutions that are natively available in Ethereum (Dinh et al., 2018). For example, a custom wallet application had to be built and shipped to support the blockchain, whereas Ethereum had many popular, publicly available wallets such as Metamask, MyEtherWallet, etc., which also supported custom cryptocurrency like Cashish. Hyperledger Fabric doesn't have any native token or cryptocurrency in the system. As such, one doesn't need to have cryptocurrency in order to use the platform. However, Cashish's entire model revolves around having a custom cryptocurrency.

Ethereum comes with a native cryptocurrency called Ether. It also provides a protocol named ERC-20 that makes it simple to build custom cryptocurrencies on the Ethereum blockchain using smart contracts (Chen, 2018; Fenu et al., 2018). Since most clinical trial participants may not necessarily have advanced technical backgrounds, it was important to choose a platform that participants could be better acquainted with. Bitcoin and Ethereum were covered by financial media outlets more often 
compared to Hyperledger. However, Bitcoin is not a DApp development framework. For these reasons, Ethereum was selected as the platform to build Cashish on.

\section{Incentives Modelling Process}

The Cashish platform program could start when cannabis companies or research companies posted requests on the Cashish portal for research participants, offering rewards in the form of Cashish Points, the custom cryptocurrency created for the platform. Users then signed up to the Cashish platform to participate in available research projects and provided information about their public Ethereum address (i.e. account number) so that rewards could be sent to them. This could be done by connecting their Metamask wallet to the portal, a feature that Metamask natively supported. Users could then search for research projects on the platform, agreeing to submit research data to receive Cashish Points and become participants of studies. The Cashish platform interfaced with Smart Contracts developed by the project team to satisfy business requirements which were publicly available on the Ethereum blockchain at a particular address. When participants completed research requirements, the action was verified by the Cashish platform. The smart contracts of the Cashish platform would then send an amount of Cashish Points as a public transaction on the Ethereum blockchain. Additional details regarding the completion of research requirements were attached to the transaction. The user could then check their Metamask wallet to see if they'd received the Cashish Points. The user could then utilize the Cashish Points obtained from research participation in an online marketplace to purchase items or services. Figure 1 demonstrates what a transfer of tokens (Cashish Points) would look like on the Cashish blockchain-based marketplace.

\section{Smart Contracts for the Marketplace Platform}

Smart Contracts were written using Solidity and deployed to the public Ethereum network. For Cashish Points, an ERC-20 contract was written and deployed to the Ethereum network. The address of this contract was used in other smart contracts to allow distribution of Cashish Points based on research participation. For the registration of new clinical trials, a smart contract was generated when researchers introduced a new clinical trial. The associated research institution purchased an amount of Cashish Points as research budget from the platform provider. The purchases were done using fiat currency (USD, CAD) or using Ethereum. To complete research requirements a smart contract that automatically transferred Cashish Points from the researcher's Ethereum account to the participant's account upon completion of a research task/survey was used. In terms of marketplace transactions, since the purchase of items from the Cashish marketplace involved the transfer of Cashish Points, smart contracts that enabled the purchase of marketplace products were developed. Figure 2 demonstrates a diagrammatic flow of how the contracts mentioned in this section help achieve the system flow and business requirements.

\section{DISCUSSION}

\section{Perception of Cryptocurrency}

During this pilot, one of the key considerations was what study participants could do with their redeemed Cashish cryptocurrency. Although cryptocurrencies began in January 2009 with the development of Bitcoin (Hileman and Rauchs, 2017), their uptake has typically been limited to technology advocates and cryptocurrency investors. The research participants did not fall into these categories, hence this study deployed a marketplace of goods so that participants could still use their Cashish Points to receive tangible benefits.

In the future, one can predict that more people will have access to cryptocurrencies and will be more inclined to use research as a means to access and acquire said virtual currencies. One example to demonstrate this growth potential is the overall market capitalization of all cryptocurrencies, which 
Figure 1. Metamask transaction of Cashish Points between two accounts

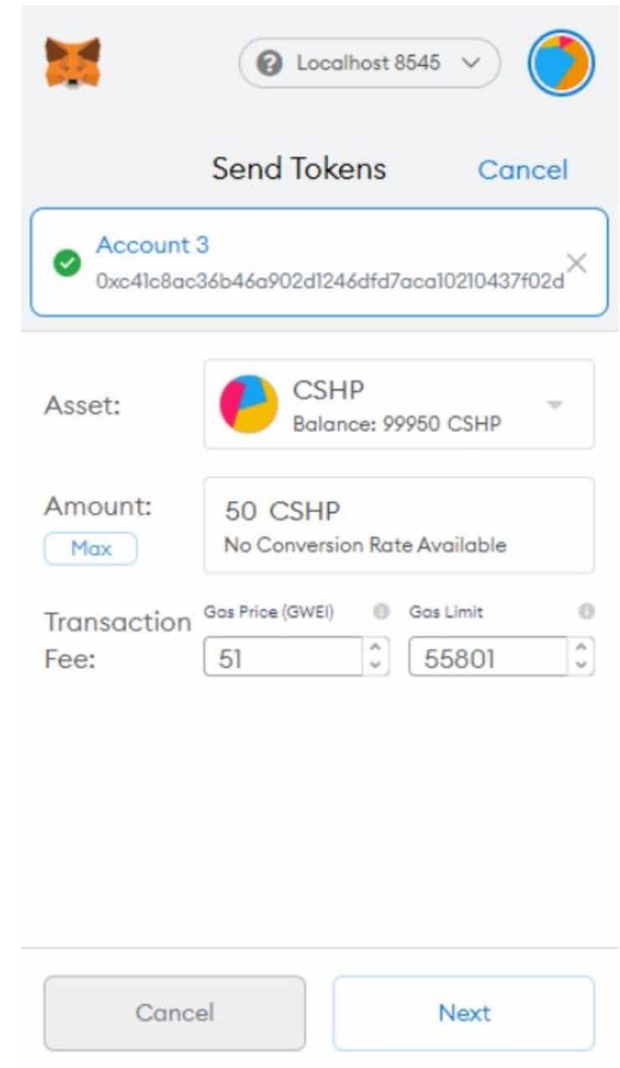

refers to the average value of currencies multiplied by the number of units available. As of 2020, the combined market cap of all cryptocurrencies sits around $\$ 334$ billion, with Bitcoin making up about $57 \%$ of the market share (Taskinsoy, 2020). This stands as a testament to the potential applicability of the electronic marketplace prototype developed in this study.

In terms of public perception of cryptocurrency, studies have employed surveys to evaluate the sentiment of the American public towards cryptocurrency, often focusing on Bitcoin as it is the most exchanged cryptocurrency (Farell, 2015). This allows conclusions made about Bitcoin to hold relatively true when extended to other cryptocurrencies. As such, surveys indicating that the majority of Americans don't know what Bitcoin is should reflect the unawareness of the average person towards cryptocurrency. Since cryptocurrencies emerged in the public eye, many retailers have sought to accept cryptocurrency as a form of payment. One example is TigerDirect, a computer and networking gear retailer. However, until more retailers accept Bitcoin, the number of users utilizing Bitcoin will be limited. Due to fear, companies such as banks have declined including cryptocurrencies in their systems. Their fears mainly revolve around one of the intrinsic properties of cryptocurrencies - a reliance on cryptographic proof as opposed to a "trust"-based system in exchanges. Circumventing this aspect of cryptocurrencies would defeat one of their sole purposes, the dissolution of a "trust"based exchange system. Other fears involved in the adoption of cryptocurrency include security, fraud, volatility, potential for tax evasion, settlement risk, and instances of bankruptcy such as that with the Mt. Gox case (Feder et al., 2017). A security breach caused currency to be lost for legitimate coin owners in said case. This has painted cryptocurrency as an insecure and untrustworthy currency unlike national currencies. Despite this, the potential functionality of cryptocurrencies in electronic 


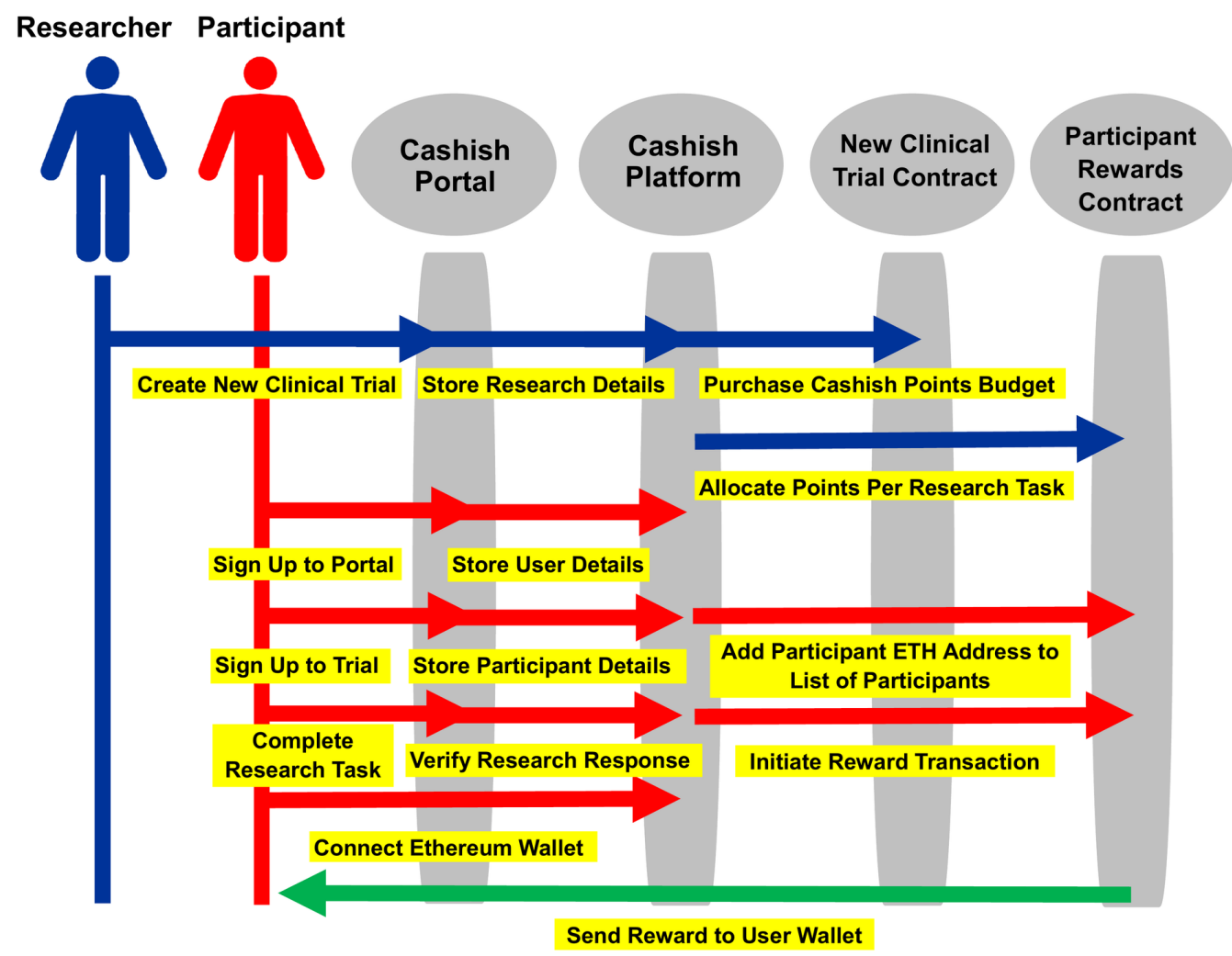

marketplaces stands to justify their presence in the Cashish platform. Cryptocurrencies function to give participants more freedom in the marketplace when it comes to redeeming the value held in their wallets.

\section{The Importance of Wallets}

To provide the users with a sense of trust, interoperability, and transparency around the virtual points they have earned, it was important that rewards were accessible outside the pilot environment. There are many steps involved in the exchange of cryptocurrency, from mining the coins to using the coins for purchase at a retailer. There are many systems in place for cryptocurrency users to navigate. One of the most important components of cryptocurrency exchange are wallets, which differ from exchanges in that while wallets function as a way to store cryptocurrency, exchanges facilitate the sale, purchase, and trading of cryptocurrency (Hileman and Rauchs, 2017). As of 2017, the number of active wallets has been estimated to be between 5.8 million and 11.5 million. Interestingly, wallet and exchanges services are becoming increasingly integrated, with roughly $50 \%$ of wallets including some sort of exchange service through their platform. Cryptocurrencies such as Bitcoin and Ethereum utilize their own blockchains for exchange (Lo and Medda, 2020). For example, Bitcoin is the central currency of the Bitcoin blockchain. On the other hand, a crypto token is a unit of value, like a cryptocurrency, that utilizes someone else's blockchain. Typically, crypto tokens utilize the Ethereum blockchain. Together, cryptocurrencies and crypto tokens can be referred to as digital assets. The potential pitfalls involved in the use of non-centralized and non-trust-based exchange systems remains a strong reason why the Ethereum platform has an advantage over Hyperledger when it comes to hosting smart contracts. The 
fact that Hyperledger requires the creation of custom wallet applications opens up potential room for error that the preexisting wallets on Ethereum such as Metamask do not risk.

\section{FUTURE RESEARCH}

More research is required to understand how blockchain can be deployed in the non-financial sector. Blockchain has been introduced into non-financial industries, applications such as Domain Name Server (DNS) control, storage of secure documents on cloud-based online storage platforms, and the music industry to allocate royalties appropriately (Nofer et al., 2017). These new areas of application have led to profound growth in blockchain utilization. As the reliance on digital and internet-based transactions increases, the demand for a validated ledger recording said exchanges exponentially increases (Crosby et al., 2016). This increased reliance on digital and internet-based platforms for ensuring the validity of currency transactions demonstrates the benefits of using blockchain for the Cashish marketplace prototype. More research is needed to understand how people perceive receiving incentives via Cashish-type token systems as opposed to receiving gift cards and cash. Follow-up research to determine the level of participants engagement using the prototype compared to traditional studies is also required. Another important area of research is to explore the usability and ease of use of such a system.

There are also research opportunities to explore how Blockchain may extend to other areas of the healthcare industry and assist in addressing long-standing problems associated with record-keeping (Zhang et al., 2018). A famous problem in the medical field is the fragmented, unstandardized, and inoperable nature of data collection technology employed in the field. Technology spanning decades is employed despite obsolescence. Record keeping is unstandardized, leading to missing and incomplete information as well as difficulty exchanging information between healthcare service providers (Zhang et al., 2018). Information pertaining to different elements of health may be held by different practitioners, and so the full picture of a patient's health is often incomplete. Patient-centered control does not reflect the reality of the current information-holding system, and so practitioners currently maintain control and ownership of the data. Blockchain would address these problems by providing a standardized, time-stamped, immutable, and simultaneously accessible and patientcontrolled ledger system (Zhang et al., 2018). With patient approval, entries can be created in the blockchain system without fear of information being tampered with, missing, or inoperable with other technological subsystems. The benefits of this system become apparent in an example using clinical trial data. Here, participants' data can be entered with assurance that the information required is present, untampered, time-stamped, and easily accessible at a later time. Blockchain technology would also assure anonymity and allow data usage only with participant control.

Another avenue which can be explored is the functionality of AI-driven tools in improving participation in clinical trials. Perhaps the step beyond digital marketplaces are curated marketplaces where participants are matched up with clinical trials via AI tools such that they are shown opportunities that would best suit their desires. This could assist in filtering through clinical trial listings so that the right participants can find adequate studies or potential new opportunities. AI tools have been used to improve multiple facets of electronic business and development, such as in banking and internet search engines (El-Gohary et al., 2021; Yuniarthe, 2017).

\section{CONCLUSION}

This paper sought to describe the benefits associated with the use of a Blockchain-based incentivization system. In this case, a prototype marketplace was developed called Cashish to bolster data collection and participant recruitment in Virtual Clinical Trials. The basis of this system relies on a marketplace for cryptocurrency exchange that is backed by researchers and can be accessed by participants. Research participants can securely sign off on the use of their data in studies to earn incentives in the 
Cashish marketplace. Built upon a Blockchain infrastructure, the prototype marketplace can securely serve to increase research engagement in an otherwise difficult area for participant recruitment. This system has the potential to benefit both participants and researchers and ultimately fulfill the need for increased participation in studies on sensitive subjects.

\section{ACKNOWLEDGMENT}

This research was funded by the Ontario Centre for Excellence (OCE) through the Advanced Technology Platforms Demonstration Program (OCE \#: 32630). 


\section{REFERENCES}

Ali, Z., Anderson, K., Chiriac, A., Andersen, A. D., Isberg, A. P., Moreno, F. G., Eiken, A., Thomsen, S. F., \& Zibert, J. R. (2020). High adherence and low dropout rate in a virtual clinical study of atopic dermatitis through weekly reward-based personalized genetic lifestyle reports. PLoS One, 15(7), e0235500. doi:10.1371/journal. pone.0235500 PMID:32614886

Almeida, S., Albuquerque, A., \& Silva, A. (2018, April). An approach to develop software that uses blockchain. In Computer Science On-line Conference (pp. 346-355). Springer. doi:10.1007/978-3-319-91186-1_36

Benchoufi, M., \& Ravaud, P. (2017). Blockchain technology for improving clinical research quality. Trials, 18(1), 1-5. doi:10.1186/s13063-017-2035-z PMID:28724395

Buterin, V. (2013). Ethereum whitepaper: A next-generation smart contract and decentralized application platform. https://ethereum.org/en/whitepaper/

Casino, F., Dasaklis, T. K., \& Patsakis, C. (2019). A systematic literature review of blockchain-based applications: Current status, classification and open issues. Telematics and Informatics, 36, 55-81. doi:10.1016/j. tele.2018.11.006

Chen, W., Zhang, T., Chen, Z., Zheng, Z., \& Lu, Y. (2020, April). Traveling the token world: A graph analysis of ethereum erc20 token ecosystem. In Proceedings of The Web Conference 2020 (pp. 1411-1421). doi: $10.1145 / 3366423.3380215$

Chen, Y. (2018). Blockchain tokens and the potential democratization of entrepreneurship and innovation. Business Horizons, 61(4), 567-575. doi:10.1016/j.bushor.2018.03.006

Crosby, M., Pattanayak, P., Verma, S., \& Kalyanaraman, V. (2016). Blockchain technology: Beyond bitcoin. Applied Innovation, 2(6-10), 71.

Di Angelo, M., \& Salzer, G. (2020, August). Tokens, types, and standards: Identification and utilization in Ethereum. In 2020 IEEE International Conference on Decentralized Applications and Infrastructures (DAPPS) (pp. 1-10). IEEE. doi:10.1109/DAPPS49028.2020.00001

Dinh, T. T. A., Liu, R., Zhang, M., Chen, G., Ooi, B. C., \& Wang, J. (2018). Untangling blockchain: A data processing view of blockchain systems. IEEE Transactions on Knowledge and Data Engineering, 30(7), 1366-1385. doi:10.1109/TKDE.2017.2781227

El-Gohary, H., Thayaseelan, A., Babatunde, S., \& El-Gohary, S. (2021). An exploratory study on the effect of Artificial Intelligence-enabled technology on customer experiences in the banking sector. Journal of Technological Advancements, 1(1), 1-17. doi:10.4018/JTA.20210101.oa1

Ethereum. (2021). ERC-20 token standard. https://ethereum.org/en/developers/docs/standards/tokens/erc-20/

Farell, R. (2015). An analysis of the cryptocurrency industry. Wharton Research Scholars, 130.

Feder, A., Gandal, N., Hamrick, J. T., \& Moore, T. (2017). The impact of DDoS and other security shocks on Bitcoin currency exchanges: Evidence from Mt. Gox. Journal of Cybersecurity, 3(2), 137-144. doi:10.1093/ cybsec/tyx 012

Fenu, G., Marchesi, L., Marchesi, M., \& Tonelli, R. (2018, March). The ICO phenomenon and its relationships with ethereum smart contract environment. In 2018 International Workshop on Blockchain Oriented Software Engineering (IWBOSE) (pp. 26-32). IEEE. doi:10.1109/IWBOSE.2018.8327568

Heath, J., Williamson, H., Williams, L., \& Harcourt, D. (2018). "It's just more personal”: Using multiple methods of qualitative data collection to facilitate participation in research focusing on sensitive subjects. Applied Nursing Research, 43, 30-35. doi:10.1016/j.apnr.2018.06.015 PMID:30220360

Hileman, G., \& Rauchs, M. (2017). Global cryptocurrency benchmarking study. Cambridge Centre for Alternative Finance, 33, 33-113.

Huynh, L., Johns, B., Liu, S. H., Vedula, S. S., Li, T., \& Puhan, M. A. (2014). Cost-effectiveness of health research study participant recruitment strategies: A systematic review. Clinical Trials, 11(5), 576-583. doi:10.1177/1740774514540371 PMID:24980278 
Kadam, R. A., Borde, S. U., Madas, S. A., Salvi, S. S., \& Limaye, S. S. (2016). Challenges in recruitment and retention of clinical trial subjects. Perspectives in Clinical Research, 7(3), 137. doi:10.4103/2229-3485.184820 PMID:27453831

Lo, Y. C., \& Medda, F. (2020). Assets on the blockchain: An empirical study of Tokenomics. Information Economics and Policy, 53, 100881. doi:10.1016/j.infoecopol.2020.100881

Macdonald, M., Liu-Thorrold, L., \& Julien, R. (2017). The blockchain: A comparison of platforms and their uses beyond bitcoin. COMS4507-Adv. Computer and Network Security.

Maese, V. A., Avery, A. W., Naftalis, B. A., Wink, S. P., \& Valdez, Y. D. (2016). Cryptocurrency: A primer. Banking LJ, 133, 468.

Maslove, D. M., Klein, J., Brohman, K., \& Martin, P. (2018). Using blockchain technology to manage clinical trials data: A proof-of-concept study. JMIR Medical Informatics, 6(4), e11949. doi:10.2196/11949 PMID:30578196

McCormack, M. (2014). Innovative sampling and participant recruitment in sexuality research. Journal of Social and Personal Relationships, 31(4), 475-481. doi:10.1177/0265407514522889

Mohanta, B. K., Panda, S. S., \& Jena, D. (2018, July). An overview of smart contract and use cases in blockchain technology. In 20189 th International Conference on Computing, Communication and Networking Technologies (ICCCNT) (pp. 1-4). IEEE. doi:10.1109/ICCCNT.2018.8494045

Monrat, A. A., Schelén, O., \& Andersson, K. (2019). A survey of blockchain from the perspectives of applications, challenges, and opportunities. IEEE Access: Practical Innovations, Open Solutions, 7, 117134-117151. doi:10.1109/ACCESS.2019.2936094

Nakamoto, S. (2008). Bitcoin: A peer-to-peer electronic cash system. Bitcoin. https://bitcoin.org/en/bitcoin-paper

National Academies of Sciences, Engineering, and Medicine. (2019). Virtual clinical trials: Challenges and opportunities: Proceedings of a workshop. Author.

Nofer, M., Gomber, P., Hinz, O., \& Schiereck, D. (2017). Blockchain. Business \& Information Systems Engineering, 59(3), 183-187. doi:10.1007/s12599-017-0467-3

Nugent, T., Upton, D., \& Cimpoesu, M. (2016). Improving data transparency in clinical trials using blockchain smart contracts. F1000 Research, 5, 2541. doi:10.12688/f1000research.9756.1 PMID:28357041

Nutt, D. J., Phillips, L. D., Barnes, M. P., Brander, B., Curran, H. V., Fayaz, A., Finn, D. P., Horsted, T., Moltke, J., Sakal, C., Sharon, H., O'Sullivan, S. E., Williams, T., Zorn, G., \& Schlag, A. K. (2021). A multicriteria decision analysis comparing pharmacotherapy for chronic neuropathic pain, including cannabinoids and cannabisbased medical products. Cannabis and Cannabinoid Research, can.2020.0129. Advance online publication. doi:10.1089/can.2020.0129 PMID:33998895

Redman, J. (2020, February 10). What are altcoins and why are there over 5,000 of them? Bitcoin.com. https:// news.bitcoin.com/altcoins-why-over-5000/

Schlag, A. K., O'Sullivan, S. E., Zafar, R. R., \& Nutt, D. J. (2021). Current controversies in medical cannabis: Recent developments in human clinical applications and potential therapeutics. Neuropharmacology, 108586, 108586. Advance online publication. doi:10.1016/j.neuropharm.2021.108586 PMID:33940011

Shrestha, A. K., \& Vassileva, J. (2018, June). Blockchain-based research data sharing framework for incentivizing the data owners. In International Conference on Blockchain (pp. 259-266). Springer. doi:10.1007/978-3-31994478-4_19

Somin, S., Gordon, G., \& Altshuler, Y. (2018, July). Network analysis of erc20 tokens trading on ethereum blockchain. In International Conference on Complex Systems (pp. 439-450). Springer. doi:10.1007/978-3-31996661-8_45

TaskinsoyJ. (2020). Bitcoin could be the first cryptocurrency to reach a market capitalization of one trillion dollars. 10.2139/ssrn.3693765

van Dorn, A. (2020). COVID-19 and readjusting clinical trials. Lancet, 396(10250), 523-524. doi:10.1016/ S0140-6736(20)31787-6 PMID:32828180 
Wang, S., \& Archer, N. P. (2007). Electronic marketplace definition and classification: Literature review and clarifications. Enterprise Information Systems, 1(1), 89-112. doi:10.1080/17517570601088380

Wong, H. H., Jessup, A., Sertkaya, A., Birkenbach, A., Berlind, A., \& Eyraud, J. (2014). Examination of clinical trial costs and barriers for drug development final. Retrieved from https://aspe.hhs.gov/system/files/pdf/77166/ rpt_erg.pdf

Yuniarthe, Y. (2017, September). Application of artificial intelligence (AI) in search engine optimization (SEO). In 2017 International conference on soft computing, intelligent system and information technology (ICSIIT) (pp. 96-101). IEEE. doi:10.1109/ICSIIT.2017.15

Zhang, P., Schmidt, D. C., White, J., \& Lenz, G. (2018). Blockchain technology use cases in healthcare. In Advances in computers (Vol. 111, pp. 1-41). Elsevier. doi:10.1016/bs.adcom.2018.03.006

Zheng, Z., Xie, S., Dai, H., Chen, X., \& Wang, H. (2017, June). An overview of blockchain technology: Architecture, consensus, and future trends. In 2017 IEEE international congress on big data (BigData congress). IEEE. doi:10.1109/BigDataCongress.2017.85

Phillip Olla (PhD) is a professor, researcher, digital health fellow, and social entrepreneur. He is currently the Chief Executive Officer of Audacia Bioscience. Dr. Olla has a PhD in Mobile Telecommunications from Brunel University in the UK. His current focus is on developing a virtual clinical trial and Al platform for transforming the research process. Dr. Olla performs a broad range of activities including designing PoC digital innovations, healthcare innovation adoption as well as designing digital health portfolios, and executive healthcare leadership training.

Mustafa Abumeeiz recently graduated from the University of Windsor with a Bachelor of Science degree and is currently working as an associate researcher for Audacia Bioscience in Windsor, Ontario.

Lauren Elliott recently graduated from the University of Windsor with a Bachelor of Science degree and is currently working as an associate researcher for Audacia Bioscience in Windsor, Ontario.

Vijay Rajasekar is a versatile software engineer. He holds a MS in Computer Science from the University of Windsor. His academic contributions involve the research of natural language generation systems and blockchain software engineering.

Stephen Bartol (MD) is a surgeon, physician executive, and entrepreneur. A long career in Orthopaedic Surgery resulted in numerous inventions and a move into the world of medical devices. He was the founder and CEO of a medical device company that was acquired by JnJ in 2017. He is currently developing new digital health tools to aid research and development of diagnostic and therapeutic choices, with a focus on COVID-19. 